\title{
Nearly chirp- and pedestal-free pulse compression in nonlinear fiber Bragg gratings
}

\author{
Qian Li, ${ }^{1}$ K. Senthilnathan, ${ }^{1}$ K. Nakkeeran, ${ }^{2}$ and P. K. A. Wai ${ }^{1, *}$ \\ ${ }^{1}$ Department of Electronic and Information Engineering, Photonics Research Center, The Hong Kong Polytechnic \\ University, Hung Hom, Hong Kong \\ ${ }^{2}$ School of Engineering, Fraser Noble Building, King's College, University of Aberdeen, Aberdeen AB24 3UE, UK \\ *Corresponding author: enwai@polyu.edu.hk
}

Received June 30, 2008; revised December 17, 2008; accepted December 17, 2008; posted December 18, 2008 (Doc. ID 98090); published February 9, 2009

\begin{abstract}
We demonstrate almost chirp- and pedestal-free optical pulse compression in a nonlinear fiber Bragg grating with exponentially decreasing dispersion. The exponential dispersion profile can be well-approximated by a few gratings with different constant dispersions. The required number of sections is proportional to the compression ratio, but inversely proportional to the initial chirp value. We propose a compact pulse compression scheme, which consists of a linear and nonlinear grating, to effectively compress both hyperbolic secant and Gaussian shaped pulses. Nearly transform-limited pulses with a negligibly small pedestal can be achieved. (C) 2009 Optical Society of America

OCIS codes: $060.3735,320.1590,320.5520$.
\end{abstract}

\section{INTRODUCTION}

Generation of short optical pulses has always been of great scientific and technological interest. There are two widely used techniques to achieve optical pulse compression; namely, higher-order soliton compression and adiabatic pulse compression [1]. The former can have a large degree of compression, but the compressed pulses suffer from significant pedestal generation, leading to nonlinear interactions between neighboring solitons. Some techniques, such as the nonlinear intensity discrimination technique $[2,3]$ can reduce the pedestals, but typically energy is wasted. Adiabatic soliton compression typically utilizes a dispersion map with monotonically decreasing dispersion along the propagation direction, $z$, and is formally equivalent to distributed amplification [4,5]. If the dispersion varies slowly enough, the soliton self-adjusts to maintain the balance between dispersion and nonlinearity (by reducing its pulse width; hence the term adiabatic compression) [6]. This compression scheme is attractive because it inherently maintains the transform-limited characteristics of the pulse as it compresses [6]. Such compression has been demonstrated experimentally by a variety of schemes to achieve the effect of decreasing dispersion [7].

As Moores pointed out in [8], exact chirped soliton solutions to the nonlinear Schrödinger (NLS) equation exist when we have distributed gain $g(z)=g_{0} /\left(1-g_{0} z\right)$ or exponentially varying dispersion $\beta_{2}(z)=\beta_{20} \exp \left(-z / z_{0}\right)$. One of the advantages of this compression scheme is that the adiabatic condition does not need to be satisfied and rapid compression is possible. More recently, a technique known as self-similar analysis has been utilized to study linearly chirped pulses in optical fibers and fiber amplifiers [9-12]. Self-similar pulses have attracted much attention since the linear chirp facilitates efficient pulse compression. In addition, these pulses can propagate without pulse breakup, called optical wave breaking, even at high powers. Kruglov et al. investigated the linearly chirped selfsimilar solitary waves in optical fiber amplifiers $[10,11]$. Billet et al. generated the linearly chirped parabolic pulses experimentally and achieved efficient pulse compression by using a hollow-core photonic bandgap (PBG) fiber [12]. However, because of the relatively small group velocity dispersion (GVD) of optical fibers, this scheme requires long fiber lengths, and only a few dispersion profiles are practically feasible. Moreover, fabrication of fibers with complex dispersion profiles usually involves the splicing of several different fibers or drawing fibers with an axially varying core diameter. A more attractive solution to achieve pulse compression is by utilizing a highly dispersive nonlinear medium such as a fiber Bragg grating (FBG). Grating dispersion just outside the stop band is up to 6 orders of magnitude larger than that of silica fiber and can be tailored simply by changing the grating profile. Grating dispersion $\left|\beta_{2}\right|$ can exceed $100 \mathrm{ps}^{2} / \mathrm{cm}$ for a fiber grating, and this feature has been used for dispersion compensation in transmission [13]. Moreover, almost any grating profiles can be manufactured using the stateof-the-art grating-writing techniques. This potential suggests utilizing this huge dispersion to construct a compact optical pulse compressor. The first experimental observation of nonlinear propagation effects in FBGs, resulting in nonlinear optical pulse compression and soliton propagation, is reported in [14]. These solitons occur at frequencies near the PBG structure of the grating, and propagate at velocities well below the speed of light in the uniform medium. Adiabatic soliton compression in nonuniform grating structures has been proposed in [15]. The adiabatic Bragg soliton compressor scheme is based on adiabatic soliton compression using a nonuniform grating in which the dispersion decreases along the grating. This technique has the advantage that it only requires one 
component (i.e., one grating) and produces a nearly pedestal-free and transform-limited pulse. Very recently, we have investigated the compression of chirped Bragg solitary pulses near the PBG structure of nonlinear FBGs (NFBGS) [16-19]. Table 1 gives the comparison between different pulse compression schemes.

In this paper, we theoretically study the linearly chirped self-similar Bragg solitary pulses near the PBG structure. Efficient pulse compression can be achieved with the appropriate grating induced dispersion. The paper is structured as follows. In Subsection 2.A, we investigate pedestal-free Bragg soliton pulse compression near the PBG structure using self-similar analysis. We discuss the physical mechanisms that determine the evolution of the chirped self-similar Bragg soliton in Subsection 2.B and we give the pulse parameter evolution equations in Subsection 2.C. In Section 3 we discuss the stepwise approximation (SWA) of the exponentially decreasing dispersion profile, and using numerical simulation we show that the compressed pulse is nearly chirp-free and almost pedestal-free. We show that the number of concatenated FBG sections is associated with the compression ratio and initial chirp value. Section 4 introduces a simple prechirper to add the required chirp profile to initial chirpfree hyperbolic secant pulse or Gaussian pulse. We find that the initial Gaussian profile evolves into a hyperbolic secant profile after compression in the NFBG. A comparison between full simulation and pulse parameter evolution equations is also given. Section 5 concludes the paper.

\section{COMPRESSION OF CHIRPED OPTICAL PULSES}

\section{A. Self-Similar Analysis}

Nonlinear pulse propagation in FBGs is governed by the nonlinear coupled mode (NLCM) equations that describe the coupling between the forward and backward traveling modes in the FBGs [1]. When the center frequency of the pulse is tuned outside but close to the PBG structure, one can apply the multiple scale analysis $[1,20,21]$ to reduce the NLCM equations into a NLS-type equation as

$$
i \frac{\partial E}{\partial z}-\frac{\beta_{2}(z)}{2} \frac{\partial^{2} E}{\partial t^{2}}+\gamma_{g}|E|^{2} E=0
$$

where $E(z, t)$ is the envelope of the Bloch wave associated with the grating, $z$ is the distance variable, $t$ is the time variable, $\beta_{2}(z)$ is the dispersion of the grating, and $\gamma_{g}$ is the effective nonlinear coefficient. Recently, we have investigated Eq. (1) to explain the formation of the chirped soliton. The Painlevé analysis to Eq. (1) implies that the soliton pulse propagation is possible only for the exponentially decreasing dispersion and constant dispersion. For the former case, we have explained the formation of chirped soliton using the variational analysis and Hirota bilinear method [22]. For Eq. (1), adiabatic Bragg soliton pulse compression has been discussed wherein the maximum compression ratio of 4 was achieved [15,21] and the pedestal generated is very small [15]. Later, using perturbation theory, Tsoy and de Sterke [23,24] have investigated the pulse compression based on the perturbed NLS equation in NFBG. Contrary to the above pulse compression studies, more recently, Rosenthal and Horowitz have proposed the pulse compression based on the reflected pulse in NFBG [25] and Bragg soliton pulse compression based on in-gap solitons [26]. In the following, we use selfsimilar analysis to show that it is possible to achieve pedestal-free compression with maximum compression ratio beyond the limit obtained by the adiabatic compression process. We assume that the self-similar solution of Eq. (1) is given by

$$
\begin{aligned}
E(z, t)= & \frac{1}{\sqrt{1-\alpha_{20} D(z)}} R\left[\frac{t-T_{c}}{1-\alpha_{20} D(z)}\right] \\
& \times \exp \left[i \alpha_{1}(z)+i \frac{\alpha_{2}(z)}{2}\left(t-T_{c}\right)^{2}\right],
\end{aligned}
$$

\begin{tabular}{|c|c|c|c|c|c|}
\hline & $\begin{array}{c}\text { Large } \\
\text { Compression } \\
\text { Ratio }\end{array}$ & Pedestal-Free & $\begin{array}{l}\text { Chirp-Free- } \\
\text { Almost } \\
\text { Chirp-Free }\end{array}$ & $\begin{array}{l}\text { Avoid Wave } \\
\text { Breaking at } \\
\text { High Powers }\end{array}$ & $\begin{array}{l}\text { Short } \\
\text { Length }\end{array}$ \\
\hline $\begin{array}{l}\text { Higher-order } \\
\text { soliton } \\
\text { compression }\end{array}$ & $\sqrt{ }$ & & & & \\
\hline $\begin{array}{c}\text { Adiabatic pulse } \\
\text { compression in } \\
\text { fibers }\end{array}$ & & & $\sqrt{ }$ & & \\
\hline $\begin{array}{c}\text { Adiabatic pulse } \\
\text { compression in } \\
\text { NFBG }\end{array}$ & & & $\sqrt{ }$ & & $\checkmark$ \\
\hline $\begin{array}{l}\text { Self-similar pulse } \\
\text { compression in } \\
\text { fibers }\end{array}$ & $\checkmark$ & $\checkmark$ & $\checkmark$ & $\checkmark$ & \\
\hline $\begin{array}{l}\text { Self-similar pulse } \\
\text { compression in } \\
\text { NFBG }\end{array}$ & $\sqrt{ }$ & $\sqrt{ }$ & $\checkmark$ & $\sqrt{ }$ & $\sqrt{ }$ \\
\hline
\end{tabular}

where

Table 1. Comparison Between Different Pulse Compression Schemes 


$$
\begin{gathered}
\alpha_{1}(z)=\alpha_{10}-\frac{\lambda_{1}}{2} \int_{0}^{z} \frac{\beta_{2}\left(z^{\prime}\right)}{\left[1-\alpha_{20} D\left(z^{\prime}\right)\right]^{2}} \mathrm{~d} z^{\prime}, \\
\alpha_{2}(z)=\frac{\alpha_{20}}{1-\alpha_{20} D(z)}, \quad D(z)=\int_{0}^{z} \beta_{2}\left(z^{\prime}\right) \mathrm{d} z^{\prime},
\end{gathered}
$$

and $\alpha_{10}, \lambda_{1}$, and $\alpha_{20}$ are integration constants and $T_{c}$ is the center of the pulse. Without loss of generality, we set the initial position of the grating $z_{0}=0$. The self-similar solution is possible if and only if the dispersion varies exponentially, i.e.,

$$
\beta_{2}(z)=\beta_{20} \exp (-\sigma z), \quad \sigma=\alpha_{20} \beta_{20},
$$

where $\beta_{20}$ is the initial dispersion value. The function $R(\theta)$ obeys the equation

$$
\frac{d^{2} R}{d \theta^{2}}-\lambda_{1} R+2 \lambda_{2} R^{3}=0
$$

where the scaling variable $\theta$ and the coefficient $\lambda_{2}$ are given by $\theta=\left(t-T_{c}\right) /\left[1-\alpha_{20} D(z)\right]$ and $\lambda_{2}=-\gamma_{g} / \beta_{20}$, respectively. Finally, the chirped bright Bragg solitary wave is given by

$$
\begin{aligned}
E(z, t)= & \sqrt{\frac{\left|\beta_{2}(z)\right|}{\gamma_{g}}} \frac{1}{T_{0}\left[1-\alpha_{20} D(z)\right]} \\
& \times \operatorname{sech}\left\{\frac{t-T_{c}}{T_{0}\left[1-\alpha_{20} D(z)\right]}\right\} \\
& \times \exp \left[i \alpha_{1}(z)+i \frac{\alpha_{2}(z)}{2}\left(t-T_{c}\right)^{2}\right],
\end{aligned}
$$

where the integration constant $\lambda_{1}$ is equal to $1 / T_{0}^{2}$, and $T_{0}$ is the initial pulse width parameter. Note that the initial full width at half-maximum (FWHM) intensity of the pulse is $1.76 T_{0}$. Equations (4) and (5) are the key results of this work, which state that efficient pedestal-free Bragg soliton pulse compression is possible using NFBG with an exponentially decreasing dispersion profile. Since the pulse width parameter varies with distance along the grating as $T(z)=T_{0} \exp (-\sigma z)$, the pulse compression ratio is $T_{0} / T(z)=\exp (\sigma z)$, which is the same as the ratio of the initial to the final dispersion. We note that while the chirp parameter $\alpha_{2}(z)=\alpha_{20} \exp (\sigma z)$, the normalized chirp value $C(z)=\alpha_{2}(z) T^{2}(z)=\alpha_{20} T_{0}^{2} \exp (-\sigma z)$. Thus as the chirped self-similar soliton compresses, the normalized chirp decreases. Consequently, the time-bandwidth product approaches 0.315 , which is the value for transform-limited hyperbolic secant pulses. Figure 1 shows the evolution of the time-bandwidth product of the compression of a selfsimilar soliton in which the initial pulse parameters are $T_{0}=10 \mathrm{ps}$ and $\alpha_{20}=-0.01 \mathrm{THz}^{2}$. The nonlinear grating has an exponentially decreasing dispersion profile with $\beta_{2}(z)$ $=\beta_{20} \exp (-\sigma z) \quad$ where initial dispersion value $\beta_{20}=-33 \mathrm{ps}^{2} / \mathrm{cm}$ [6] and the dispersion decay rate $\sigma$ $=0.33 / \mathrm{cm}$. The nonlinear coefficient of the grating $\gamma_{g}$ $=15 \mathrm{~W} / \mathrm{km}$. The time-bandwidth product decreases from 0.761 to 0.327 after the soliton travels $8 \mathrm{~cm}$ of the nonlinear grating.

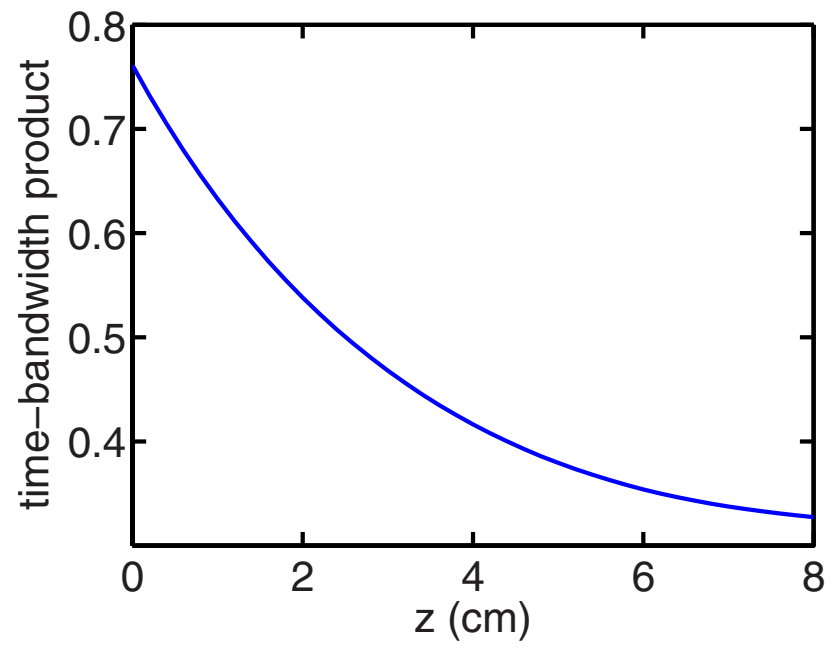

Fig. 1. (Color online) Evolution of the time-bandwidth product of a self-similar chirped soliton. The initial pulse parameters are $T_{0}=10 \mathrm{ps}$ and $\alpha_{20}=-0.01 \mathrm{THz}^{2}$. The grating parameters are $\beta_{20}$ $=-33 \mathrm{ps}^{2} / \mathrm{cm}, \sigma=0.33 / \mathrm{cm}$, and $\gamma_{g}=15 \mathrm{~W} / \mathrm{km}$.

\section{B. Evolution of the Self-Similar Soliton}

In this section we will study how the physical mechanisms determine the evolution of the self-similar soliton of Eq. (5) as the grating and pulse parameters vary. Since the soliton is chirped, the soliton evolution depends on the interaction between the pulse chirp and the grating dispersion and also between the grating dispersion and the nonlinearity. The effect of dispersion and nonlinearity can be measured by the distance-dependent dispersion and nonlinear lengths, which are defined, respectively, as $L_{D}(z)=T^{2}(z) /\left|\beta_{2}(z)\right|$ and $L_{N}(z)=1 / \gamma_{g}(z) / P(z)$, where $P(z)$ is the peak intensity of the optical pulse. The dispersion length is defined for a chirp-free pulse. To account for the effect of the pulse chirp, we define a chirp length in analogous to the dispersion length as $L_{C}(z)$ $=T^{2}(z) /|C(z)| /\left|\beta_{2}(z)\right|$. For a linear medium governed by GVD only, the dispersion length and the chirp length completely characterize the evolution of a chirped pulse. For the self-similar soliton in Eq. (5), we have $L_{D}(z)=L_{N}(z)$. We also find that the chirp length $L_{C}=1 / \sigma$, which is the characteristic length of the exponentially varying dispersion. Thus the self-similar soliton evolves in such a way that the dispersion length always equals the nonlinear length while the chirp length remains constant.

The evolution of the self-similar soliton depends on the relative strength of the chirp length and the dispersion length. For ease of discussion, we assume in the following that the dispersion is exponentially decreasing, i.e., $\sigma$ $>0$. Since the nonlinear length or dispersion length is exponentially decreasing, even if the effect of chirp dominates initially, i.e., $L_{N} \gg L_{C}$ at $z=0$, the nonlinear effect will eventually become dominant as the distance becomes very large; i.e., $L_{N} \ll L_{C}$ as $z \rightarrow \infty$. When the effect of chirp is large, the self-similar soliton evolves quasi-linearly. The compression of the soliton is mainly due to the unwinding of the pulse chirp by the grating dispersion. When the effect of chirp is small, the soliton evolves nonlinearly. The soliton compression is mainly due to the interplay between the dispersion effect and the self-phase modulation. Thus the physical mechanism governing the 
compression of the self-similar soliton depends on the initial grating parameters. Note that the initial soliton chirp coefficient $\alpha_{20}=\sigma / \beta_{20}$, where $\beta_{20} / \sigma$ is the total dispersion of the NFBG with infinite length. The total dispersion of the NFBG with length $L$ is $S(L)=\left(\beta_{20} / \sigma\right)[1-\exp (-\sigma L)]$. Thus if $L_{N} \gg L_{C}$ at $z=0$, the soliton will evolve quasilinearly in the beginning of the grating, then evolve according to a combination of linear and nonlinear effects around intermediate distance, and finally, nonlinearly for large value of $z$. If $L_{N} \sim L_{C}$ or $L_{N} \ll L_{C}$ at $z=0$, we will only observe part of the transition from quasi-linear evolution to full nonlinear evolution. To demonstrate that the soliton evolves quasi-linearly when $L_{N} \gg L_{C}$, we consider a distance $z_{0}$ such that $\varepsilon \equiv L_{C} / L_{N}\left(z_{0}\right) \ll 1$. We then normalize Eq. (1) as

$$
i \frac{\partial U}{\partial \zeta}-\frac{\operatorname{sgn}\left(\beta_{20}\right) f(\zeta)}{2} \frac{\partial^{2} U}{\partial \tau^{2}}+|U|^{2} U=0,
$$

where $\zeta=z / L_{D 0}, \tau=\left(t-T_{c}\right) / T_{0}$, and $U=E \sqrt{\gamma_{g} L_{N 0}}$ are the normalized space, time, and electric field, respectively. The parameters $L_{D 0}$ and $L_{N 0}$ are the dispersion and nonlinear lengths at $z=z_{0}$. The function $f(\zeta)=\beta_{2}(z) / \beta_{20}$ $=\exp (-\zeta / \varepsilon)$ is a rapidly varying function of $\zeta$. We also note that the quadratic phase in Eq. (5) in normalized variables is given by $\phi=\eta(\zeta)+\exp (\zeta / \varepsilon) \tau^{2} / 2 / \varepsilon$, where $\eta(\zeta)$ $=\alpha_{1}\left(L_{D 0} \zeta\right)$. Thus the quadratic phase of the soliton is a large and rapidly varying function of $\zeta$. We use the multiple length scale expansion to determine the evolution of the chirped Bragg soliton under a rapidly varying dispersion and large and rapidly varying chirp. We introduce the fast and slow space and time variables as

$$
\begin{aligned}
& \zeta_{1}=\zeta / \varepsilon, \quad \tau_{1}=\tau / \varepsilon, \\
& \zeta_{2}=\zeta, \quad \tau_{2}=\tau,
\end{aligned}
$$

where the subscripts 1 and 2 represent the fast and slow variables, respectively. The normalized pulse is now a function of both fast and slow variables, i.e., $U\left(\zeta_{1}, \zeta_{2}, \tau_{1}, \tau_{2}\right)$. We then expand the normalized electric field $U\left(\zeta_{1}, \zeta_{2}, \tau_{1}, \tau_{2}\right)$ in terms of the small parameter $\varepsilon$ as

$$
\begin{aligned}
U\left(\zeta_{1}, \zeta_{2}, \tau_{1}, \tau_{2}\right)= & U^{(0)}\left(\zeta_{1}, \zeta_{2}, \tau_{1}, \tau_{2}\right)+\varepsilon U^{(1)}\left(\zeta_{1}, \zeta_{2}, \tau_{1}, \tau_{2}\right)+\varepsilon^{2} U^{(2)} \\
& \times\left(\zeta_{1}, \zeta_{2}, \tau_{1}, \tau_{2}\right)+\ldots,
\end{aligned}
$$

where $U^{(j)}\left(\zeta_{1}, \zeta_{2}, \tau_{1}, \tau_{2}\right), j=0,1,2, \ldots, \infty$, is the $j$ th order expansion of $U\left(\zeta_{1}, \zeta_{2}, \tau_{1}, \tau_{2}\right)$. Finally, through the substitution of Eqs. (7) and (8) into Eq. (6) and at order $1 / \varepsilon$, we obtain

$$
i \frac{\partial V^{(0)}}{\partial \zeta_{1}}-\frac{1}{2} \operatorname{sgn}\left(\beta_{20}\right) \exp \left(-\zeta_{1}\right) \frac{\partial^{2} V^{(0)}}{\partial \tau_{1}{ }^{2}}=0,
$$

where we further assume a separation of variables $U^{(0)}$ $\times\left(\zeta_{1}, \zeta_{2}, \tau_{1}, \tau_{2}\right)=V^{(0)}\left(\zeta_{1}, \tau_{1}\right) W^{(0)}\left(\zeta_{2}, \tau_{2}\right)$ because the coefficient in Eq. (9) depends on the fast variable only. The function $V^{(0)}\left(\zeta_{1}, \tau_{1}\right)$ depends on the fast variables only while $W^{(0)}\left(\zeta_{2}, \tau_{2}\right)$ depends on the slow variables only. Thus from Eq. (9), when $\varepsilon=L_{C} / L_{N}\left(z_{0}\right) \ll 1$, the fast behavior of the soliton is governed by the grating dispersion only. The nonlinear effect affects the soliton evolution at slow space and time scales only. The above analysis demonstrates that the self-similar chirped soliton evolves quasi-linearly when $L_{N} \gg L_{C}$. As an example, Fig. 2(a) shows the evolution of the FWHM of the soliton in which $T_{0}=80 \mathrm{ps}$ and $\alpha_{20}=-0.01 \mathrm{THz}^{2}$. The grating parameters are $\beta_{20}=$ $-33 \mathrm{ps}^{2} / \mathrm{cm}$ and $\sigma=0.33 / \mathrm{cm}$. The dots and solid curve represent the FWHM with and without the nonlinearity. Initially at $z=0, L_{D} \sim 194 \mathrm{~cm}, L_{C} \sim 3 \mathrm{~cm}, \varepsilon \sim 0.0156$. At grating length of $6 \mathrm{~cm}, L_{D} \sim 26.8 \mathrm{~cm}, L_{C} \sim 3 \mathrm{~cm}, \varepsilon \sim 0.11$. We observe that the evolution of the FWHM of the soliton is well-approximated by the linear evolution. Figure 2(b) plots the evolution of the FWHM of an initial chirp-free hyperbolic secant pulse with the same parameters as the chirped soliton in Fig. 2(a) except that $\alpha_{20}=0$. The dots and solid curve represent the FWHM with and without the nonlinearity. We observe that the pulse width does not vary significantly and the linear evolution does not agree with the nonlinear evolution at all, thus demonstrating the importance of the chirp in the self-similar chirped soliton evolution.

Finally if $L_{N} \ll L_{C}$ at $z=0$, then grating dispersion $\beta_{2}(z)=\beta_{20} \exp (-\sigma z)$ varies slowly and the normalized chirp $C(z)=C_{0} \exp (-\sigma z)$ is very small. Hence in the distance on the order of $L_{N}$, the pulse evolution is governed mainly by the interaction between the dispersion and the nonlinearity. The chirp affects the pulse evolution only at distance on the order of $L_{C}$.

\section{Pulse Parameter Evolution Equations}

Equation (5) is the exact soliton solution to Eq. (1). To determine the pulse compression when the grating dispersion profile is not exponentially varying or the initial pulse deviates from the self-similar soliton, we can either numerically simulate Eq. (1) directly or use semianalytical reduction methods such as the Lagrangian variational method (LVM) [27] or the projection operator method (POM) $[28,29]$ to derive the equations governing the evolution of the pulse parameters. In both the LVM and the POM, the success of the approximation depends on the ansatz chosen. For the NLS-type equation like Eq. (1), both hyperbolic secant pulse shape [Eq. (10)] and Gaussian pulse shape [Eq. (11)] with a quadratic phase variation are common ansatz, i.e.,

$$
E=x_{1} \operatorname{sech}\left(\frac{t}{x_{2}}\right) \exp \left(\frac{i x_{3} t^{2}}{2}+i x_{4}\right),
$$

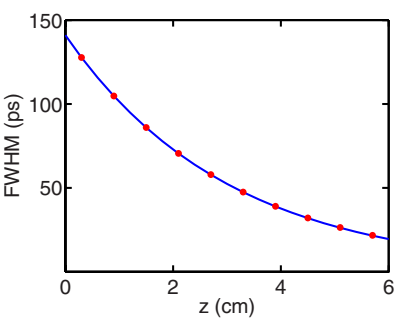

(a)

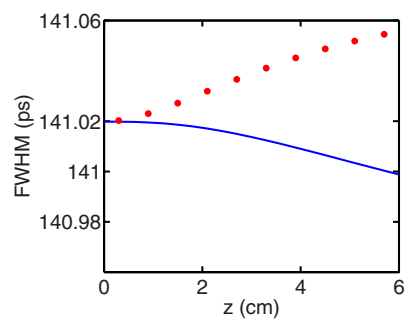

(b)
Fig. 2. (Color online) Evolution of the FWHM of (a) chirped hyperbolic secant pulse $\left(T_{0}=80 \mathrm{ps}, \alpha_{20}=-0.01 \mathrm{THz}^{2}\right)$ and (b) unchirped hyperbolic secant pulse $\left(T_{0}=80 \mathrm{ps}, \alpha_{20}=0\right)$ in exponentially decreasing dispersion $\left(\beta_{20}=-33 \mathrm{ps}^{2} / \mathrm{cm}, \sigma=0.33 / \mathrm{cm}\right)$. The dots and solid curve represent the evolution with $\left(\gamma_{g}\right.$ $=15 \mathrm{~W} / \mathrm{km}$ ) and without nonlinearity, respectively. 


$$
E=x_{1} \exp \left(-\frac{t^{2}}{x_{2}{ }^{2}}+\frac{i x_{3} t^{2}}{2}+i x_{4}\right),
$$

where $x_{\mathrm{i}}(z), i=1,2,3,4$ are the amplitude, pulse width, chirp, and phase parameters of the optical pulse. Equations [12] give the equations governing the evolution of the pulse parameters generated by the LVM and the POM using the hyperbolic secant pulse and Gaussian pulse ansatz. The coefficients $c_{i}, i=1,2,3,4$ are constants independent of distance $z$ but depend on the reduction method and the ansatz used. In other words, the pulse parameters equations resulting from the two reduction methods and two different pulse ansatz have the same functional form. Only some of the coefficients in the equations are different,

$$
\begin{aligned}
& \frac{\mathrm{d} x_{1}}{\mathrm{~d} z}=\frac{1}{2} \beta_{2}(z) x_{1} x_{3}, \\
& \frac{\mathrm{d} x_{2}}{\mathrm{~d} z}=-\beta_{2}(z) x_{2} x_{3}, \\
& \frac{\mathrm{d} x_{3}}{\mathrm{~d} z}=\beta_{2}(z)\left(x_{3}{ }^{2}-c_{1} \frac{1}{x_{2}{ }^{4}}\right)-c_{2} \frac{\gamma_{g} x_{1}{ }^{2}}{x_{2}{ }^{2}}, \\
& \frac{\mathrm{d} x_{4}}{\mathrm{~d} z}=c_{3} \frac{\beta_{2}(z)}{x_{2}{ }^{2}}+c_{4} \gamma_{g} x_{1}{ }^{2} .
\end{aligned}
$$

Table 2 lists the values of the constant $c_{i}$ 's for different combinations of the reduction methods and ansatz used.

In general, the solutions to the pulse parameter equations are only the "best" approximation to the solution to the original equations under the assumption of the ansatz and the reduction method used. However, if the functional form of the ansatz coincides with that of the exact solution, as in the case of the ansatz Eq. (10) to Eq. (1), then the pulse parameter equations will yield the exact solution to the original equations. Using the LVM and the hyperbolic secant pulse as ansatz, the first three equations in Eq. (12) can be written as

$$
\begin{aligned}
& \frac{\mathrm{d} x_{1}}{\mathrm{~d} z}=\frac{x_{1}}{2 L_{C}(z)}, \\
& \frac{\mathrm{d} x_{2}}{\mathrm{~d} z}=-\frac{x_{2}}{L_{C}(z)},
\end{aligned}
$$

$$
\frac{\mathrm{d} x_{3}}{\mathrm{~d} z}=\frac{x_{3}}{L_{C}(z)}+\frac{4}{\pi^{2} x_{2}{ }^{2}}\left[\frac{1}{L_{D}(z)}-\frac{1}{L_{N}(z)}\right]
$$

where $\quad L_{C}(z)=1 /\left(x_{3} \beta_{2}\right), \quad L_{D}(z)=-x_{2}^{2} / \beta_{2}, \quad$ and $\quad L_{N}(z)$ $=1 /\left(\gamma_{g} x_{1}^{2}\right)$ represent the chirp, dispersion, and nonlinear lengths, respectively. Equation (13) can be solved readily if we assume $L_{D}(z)=L_{N}(z)$, i.e., the well-known condition in which the dispersion length equals the nonlinear length. The solutions are

$$
\begin{aligned}
& x_{1}(z)=x_{1}(0) \exp \left(\frac{1}{2} \int_{0}^{z} 1 / L_{C}\left(z^{\prime}\right) \mathrm{d} z^{\prime}\right), \\
& x_{2}(z)=x_{2}(0) \exp \left(-\int_{0}^{z} 1 / L_{C}\left(z^{\prime}\right) \mathrm{d} z^{\prime}\right), \\
& x_{3}(z)=x_{3}(0) \exp \left(\int_{0}^{z} 1 / L_{C}\left(z^{\prime}\right) \mathrm{d} z^{\prime}\right) .
\end{aligned}
$$

It is then necessary to substitute $x_{1}(z), x_{2}(z)$, and $x_{3}(z)$ back into the condition $L_{D}(z)=L_{N}(z)$ to check for the consistency. There are two possibilities for the parameter $L_{C}$ that needed to be treated separately. First, we consider $L_{C} \rightarrow \infty$, i.e., $x_{3} \beta_{2}=0$, which means $x_{3}(z)=0$ since $\beta(z) \neq 0$. In this case $x_{1}, x_{2}$, and $x_{3}$ are independent of $z$. The condition $L_{D}(z)=L_{N}(z)$ means that $\beta_{2}(z)=\beta_{20}$ is a constant and the parameters $x_{1}$ and $x_{2}$ satisfy the condition $-x_{2}^{2} / \beta_{20}=1 /\left(\gamma_{g} x_{1}^{2}\right)$. In other words, we obtain the conventional soliton solutions for the NLS equation with constant dispersion. Next, we consider the case when $L_{C}$ is finite. Thus $\beta_{2}(z)=\left[1 / x_{3}(0) / L_{C}(z)\right] \exp \left(-\int_{0}^{z} 1 / L_{C}\left(z^{\prime}\right) \mathrm{d} z^{\prime}\right)$. The condition $L_{D}(z)=L_{N}(z)$, which becomes $-x_{2}(0)^{2} x_{3}(0) L_{C}(z)=1 /\left[\gamma_{g} x_{1}(0)^{2}\right]$, can be satisfied if and only if $L_{C}$ is a constant independent of $z$. As a result $x_{1}(z)$, $x_{2}(z), x_{3}(z)$, and $\beta_{2}(z)$ vary exponentially with distance $z$, which is the chirped soliton solution for the NLS equation with exponentially varying dispersion. In summary, we

\begin{tabular}{|c|c|c|c|c|c|}
\hline Method & Ansatz & $c_{1}$ & $c_{2}$ & $c_{3}$ & $c_{4}$ \\
\hline \multirow[t]{2}{*}{$\operatorname{LVM}^{a}$} & Hyperbolic & 4 & 4 & 1 & 5 \\
\hline & secant & $\overline{\pi^{2}}$ & $\overline{\pi^{2}}$ & $\overline{3}$ & $\overline{6}$ \\
\hline \multirow{2}{*}{$\mathrm{POM}^{b}$} & Hyperbolic & 30 & 30 & $\begin{array}{ll}1 & 5\end{array}$ & $\left(\begin{array}{ll}2 & 5\end{array}\right.$ \\
\hline & secant & $\overline{\pi^{4}}$ & $\overline{\pi^{4}}$ & $\left(\overline{6}+\overline{4 \pi^{2}}\right)$ & $\overline{3}+\overline{4 \pi^{2}}$ \\
\hline \multirow[t]{2}{*}{ LVM-POM } & Gaussian & 4 & $\sqrt{2}$ & 1 & 5 \\
\hline & & & & & $\overline{4 \sqrt{2}}$ \\
\hline
\end{tabular}
observe that the soliton solutions for the NLS equation with the constant dispersion and exponentially varying dispersion share the same characteristics; both of them evolve such that the dispersion length equals the nonlinear length and the chirp length remains unchanged.

In the following, we will use the pulse parameter equations [Eq. (12)] as well as full numerical simulation of the NLS equation [Eq. (1)] to study the self-similar optical pulse compression.

Table 2. Values of the Constants $c_{i}$ in Eq. (12) for Different Choices of the Reduction Methods and Ansatz

${ }^{a}$ LVM-Lagrangian variational method.

${ }^{b}$ POM-Projection operator method. 


\section{STEPWISE APPROXIMATION}

The self-similar soliton requires the grating dispersion to vary exponentially. Although almost any grating profile can be manufactured using the state-of-the-art gratingwriting techniques, fabrication of FBGs with an exact exponentially decreasing dispersion profile for an extended length is still challenging, especially when the grating length $L$ is significantly larger than the characteristic length of the grating $L_{C}=1 / \sigma$. Similar problems have been encountered in utilizing dispersion decreasing fibers to reduce pulse broadening or the nonadiabatic effects caused by lumped amplifiers in fiber soliton transmission systems. Hasegawa et al. have shown that a stepwise dispersion-decreasing fiber can reduce the collisioninduced jitters in soliton-based wavelength-division multiplexing systems [30] when the number of steps is sufficiently large (four or more). Therefore, in this section we will study the feasibility of using the stepwise constant function to approximate the exponentially decreasing dispersion profile and determine the effects of the SWA on the quality of the compressed pulse. SWA could be easily realized by concatenating FBGs with different uniform dispersions together. We assume that a nonuniform grating is divided into a number of sections with equal lengths. We then determine the total dispersion in each section of gratings and replace each section with a uniform grating with the corresponding total dispersion value. If $M$ sections are used, the constant dispersion value $b_{i}$ for the $i$ th section is given by

$$
b_{i}=\frac{M}{L} \int_{(i-1)(L / M)}^{i(L / M)} \beta_{20} \exp \left(-\alpha_{20} \beta_{20} z^{\prime}\right) \mathrm{d} z^{\prime} .
$$

From Subsection 2.B only one grating with constant dispersion will be sufficient to well approximate the pulse compression in the chirp dominated regime, i.e., $L_{C} \ll L_{N}$. In this case, the pulse evolves quasi-linearly as shown in Fig. 2. The pulse compression is determined by the total dispersion in the grating, and the detail of the dispersion profile is not important. This regime is obviously not of interest. Similarly, we are not interested in the nonlinear regime in which $L_{C} \gg L_{N}$, because the chirp is not playing an important role unless the grating length is of the order of $L_{C}$. We therefore will focus on pulse compression when $L_{C} \sim L_{N}$ and study both dispersion exponentially decreasing FBG (DDFBG) and the SWA to the dispersion profile.

As an example, we consider a grating length of $L_{g}$ $=6 \mathrm{~cm}$; the initial grating dispersion is $\beta_{20}=-33 \mathrm{ps}^{2} / \mathrm{cm}$ and the decay rate $\sigma=0.33 \mathrm{~cm}$. Thus the grating length is almost twice the characteristic length of the grating. We assume an initial pulse width parameter $T_{0}=10 \mathrm{ps}$, and the initial chirp coefficient $\alpha_{20}=-0.01 \mathrm{THz}^{2}$. The initial dispersion length $L_{D 0} \sim 3 \mathrm{~cm}$. Hence initially $L_{D 0}=L_{C}$. At the end of the grating, the dispersion decreases to $\beta_{2}(L)$ $\sim-4.56 \mathrm{ps}^{2} / \mathrm{cm}$ and $L_{D}\left(L_{g}\right) \sim 0.14 L_{C}$. Figure 3(a) shows the exponentially decreasing dispersion profile (dashed curve) and the six-section SWA (solid curve). Figure 3(b) shows the initial pulse profile (dotted-dashed curve) and the compressed pulse using the DDFBG (solid curve) and the six-section SWA (dashed curve). The compression ratio is $\sim 7$ in both cases. A small pedestal (1.12\%) appears in the SWA profile. We calculate the pedestal using the

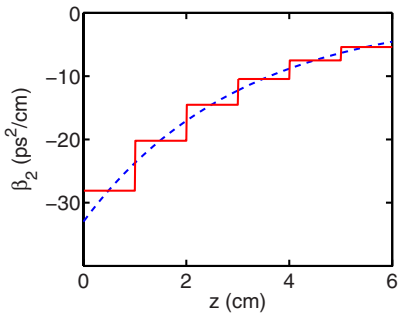

(a)

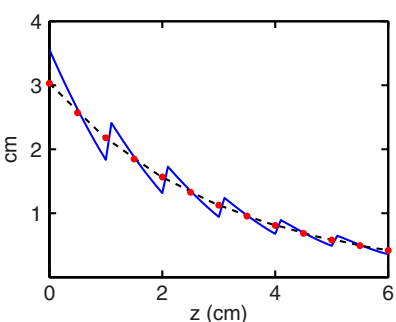

(c)

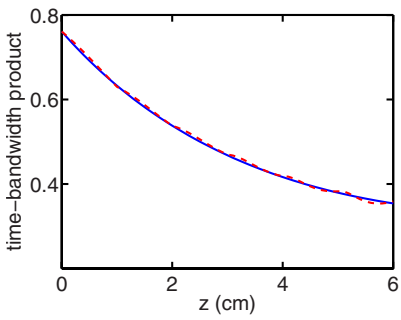

(e)

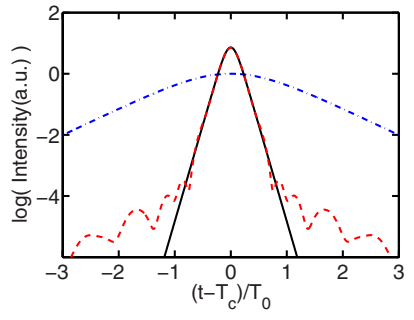

(b)

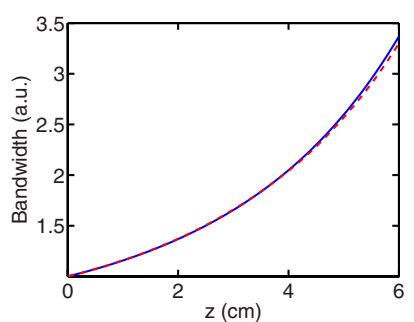

(d)

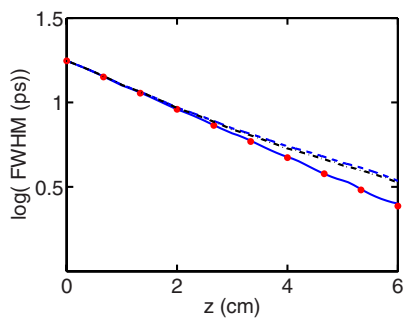

(f)
Fig. 3. (Color online) (a) Exponentially decreasing dispersion profile (dashed curve) and the six-section SWA (solid curve). (b) The pulse profile at $z=0$ (dotted-dashed curve) and at $z=6 \mathrm{~cm}$ in the DDFBG (solid curve) and the six-section SWA (dashed curve). (c) Evolutions of the dispersion length $L_{D}$ and nonlinear length $L_{N}$. The dots, dashed curve, and solid curve represent $L_{D}\left(=L_{N}\right)$ in DDFBG, $L_{N}$ in SWA, and $L_{D}$ in SWA, respectively. (d) Bandwidth broadening in DDFBG (solid curve) and the six-section SWA (dashed curve). (e) Time-bandwidth product for DDFBG (solid curve) and the six-section SWA (dashed curve). (f) Evolution of FWHM in the six-section SWA for the self-similar soliton (dots), the full numerical simulations (solid curve), the LVM with hyperbolic secant pulse ansatz (dashed curve), and the POM with hyperbolic secant pulse ansatz (dotted-dashed curve).

method described in [31]. Figure 3(c) shows the evolution of the dispersion length $\left(L_{D}\right)$ and nonlinear length $\left(L_{N}\right)$. In DDFBG, $L_{D}$ is always equal to $L_{N}$ (dots). The nonlinear length of the SWA (dashed curve) agrees with that of exponentially decreasing dispersion profile while the dispersion length of the SWA (solid curve) shows discontinued jumps at the boundaries of each grating section. From Fig. 3(c), it is obvious that the dispersion length $L_{D}$ of the SWA approximates the nonlinear length $L_{N}$ very well, showing that the area under the solid curve is almost equal to the area under the dashed curve. Figures 3(d) and 3(e) give the evolutions of bandwidth and timebandwidth product during pulse compression for both dispersion profiles, respectively. The solid and dashed curves represent the results from the DDFBG and the six-section SWA, respectively. At the end of the grating the timebandwidth product is 0.354 for the DDFBG and 0.359 for the six-section SWA. Thus, the compressed pulse becomes almost transform limited. We use the fourth-order Runge-Kutta (RK) method to solve Eq. (12) using the hy- 
perbolic secant profile as ansatz. Figure 3(f) shows the evolution of the FWHM in the six-section SWA in the logarithmic scale, where the dots, solid curve, dashed curve, and dotted-dashed curve represent the self-similar soliton, the simulation results of Eq. (1) using the splitstep Fourier method, the results of the LVM using the hyperbolic secant pulse ansatz, and the results of the POM using the hyperbolic secant pulse ansatz, respectively. The results from the LVM and the POM are similar and both approximate the exact solution very well.

For pulse compression, it is important to determine the number of sections required to approximate the required exponentially decreasing dispersion. The number of sections required obviously depends on the tolerance of the pedestal generated. The tighter the tolerance, the higher the number of sections required. By varying the initial dispersion coefficient $\left(\beta_{20}\right)$, the decay rate of the exponentially decreasing dispersion $(\sigma)$, the compression ratio (CR) and the initial pulse width, we find that the number of sections required only depends on the compression ratio $\left[\mathrm{CR}=\exp \left(\sigma L_{g}\right)\right]$ and the ratio of initial chirp length to initial dispersion length $\left(\varepsilon=L_{C} / L_{D 0}=1 / \alpha_{20} / T_{0}^{2}=1 / C_{0}\right.$, where $C_{0}$ is the initial normalized chirp value). If $\varepsilon$ is the same for different pulses, it is obvious that the one with a larger $\mathrm{CR}$ requires more sections. If $\mathrm{CR}$ is fixed, the one with larger $\varepsilon$ requires more sections. In Fig. 4(a), the solid curve and dots represent $\varepsilon=1 / 4$ and $1 / 8$, respectively. The tolerance of the pedestal energy is $<0.5 \%$ when we implement the SWA. From Fig. 4(a), if the objective is to reduce the number of constant segments, then $\varepsilon$ should be kept small but, of course, $\varepsilon$ cannot be too small otherwise we will be in the chirp dominated regime. The chirp dominated regime is not of interest because there is no significant bandwidth broadening. Figure 4(b) shows the dependence of pulse bandwidth on the pulse chirp for a chirped Gaussian pulse of the form $A_{0} \exp \left[-(1-i C) t^{2} / T_{0}^{2} / 2\right]$ (solid curve) and chirped hyperbolic secant pulse of the form $A_{0} \operatorname{sech}\left(t / T_{0}\right) \exp \left(i C t^{2} / T_{0}^{2} / 2\right)$ (dashed curve). We observed that the ratio of the bandwidth of the chirped pulse to that of the unchirped pulse $[\Delta \omega(C) / \Delta \omega(0)]$ increases linearly and quadratically with the chirp $(C)$ for the hyperbolic secant pulse and Gaussian pulse, respectively. The bandwidth contributed by the chirp equals that of an unchirped pulse occur at $|C|=0.87$ for the Gaussian pulse and 0.36 for the hyperbolic secant pulse. Beyond the respective chirp values, the chirp contributes more significantly to the pulse bandwidth.

\section{COMPRESSION OF UNCHIRPED OPTICAL PULSES}

Unlike conventional solitons of the NLS equation with constant dispersion, the solution to the NLS equation with exponentially varying dispersion is chirped. The linear chirp of the solution is a key feature of the self-similar characteristics of the chirped soliton solution which allows pedestal-free pulse compression. However in pulse compression, typically the input pulse is unchirped although the pulse after compression is usually chirped. Thus for the proposed nonlinear pulse compression, the input pulse must be chirped in the prescribed manner before pulse compression in the nonlinear grating can take
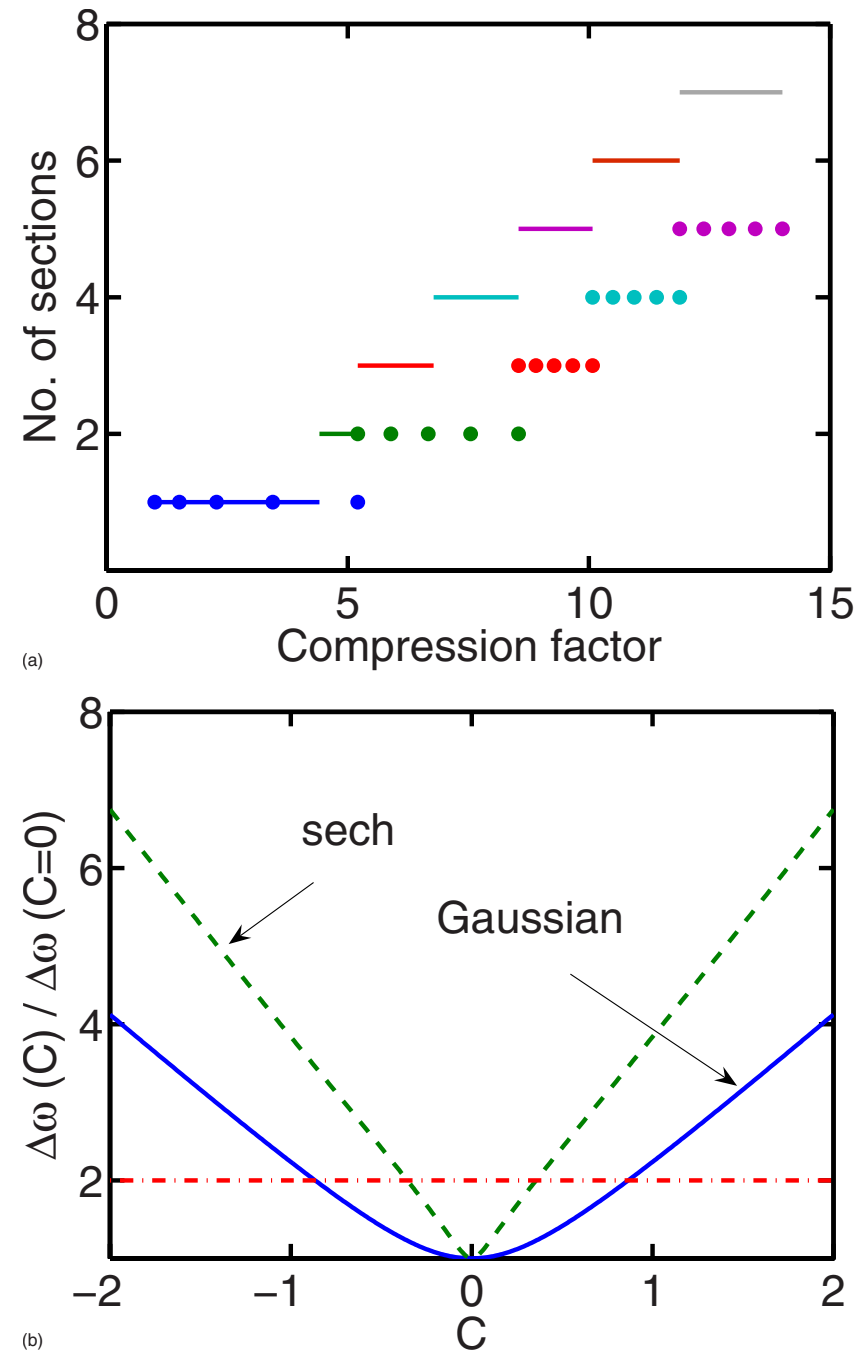

Fig. 4. (Color online) (a) Number of sections versus the compression ratio for $\varepsilon=1 / 4$ (solid curve) and $\varepsilon=1 / 8$ (dots). The tolerance of the pedestal energy is $<0.5 \%$. (b) Ratio of the bandwidth of chirped pulses versus that of unchirped pulses for Gaussian pulses (solid curve) and hyperbolic secant pulses (dashed curve). The dotted-dashed curve represents the contribution of the chirp to the bandwidth equals to that of an unchirped pulse.

place. In general, it is not easy to produce the precise pulse shape and chirp [32]. Therefore in this section we study the compression of initially chirp-free pulse using a linear FBG to produce the required chirp profile for the compression in the NFBG with exponentially decreasing dispersion. In particular, we assume that the input pulse is a chirp-free hyperbolic secant pulse or Gaussian pulse. Our simulation results show that the pedestal generated from an input Gaussian pulse is much smaller than that of an input hyperbolic secant pulse showing that the compression by the NFBG is more sensitive to the chirp profile than the pulse shape. We also find that an initial Gaussian pulse evolves into a hyperbolic secant pulse after the compression in the NFBG.

\section{A. Prechirping}

First, we study the chirping of an initially chirp-free hyperbolic secant pulse of the form, $A_{0} \operatorname{sech}\left(t / T_{0}\right)$, by using a 
linear FBG with normal dispersion, where $A_{0}$ is the pulse amplitude. Here normal dispersive medium is used to introduce a negative chirp $\alpha_{20}$ for the subsequent nonlinear pulse compression. Figure 5(a) shows the evolution of generated pedestal with the length of the linear FBG. The pedestal energy increases with the length of the linear FBG. Figure 5(b) shows the evolution of the normalized chirp $C_{\text {lin }}(z)$ in a linear FBG. The chirp value is determined by carrying out a polynomial fit of the pulse phase. We find that the higher-order chirp terms are negligible. The normalized chirp $\left|C_{\text {lin }}(z)\right|$ initially increases and then decreases with the length of the linear FBG. We note that the normalized chirp $C_{\operatorname{lin}}(z)=\alpha_{2}(z) T_{0}^{2}$ and $\alpha_{2}(z)$ is associated with the decay rate of NFBG $\sigma=\alpha_{20} \beta_{20}$. Thus for a compact optical pulse compressor, we choose the length of the linear grating to maximize the value of $\left|\alpha_{2}(z)\right|$. From Fig. 5(b) the maximum $\left|C_{\text {lin }}(z)\right|$ occurs at $z / L_{D}=0.56$ with $C_{\text {lin }}(z)=-0.3992$.

For the chirping of an initially chirp-free Gaussian pulses of the form $A_{0} \exp \left(-t^{2} / T_{0}{ }^{2} / 2\right)$, it is well-known that linear dispersive media only modify the quadratic chirp coefficient of a Gaussian pulse but leave the pulse shape unchanged. The chirp coefficent $\alpha_{20}$ is given by $\alpha_{20}=$ $-\beta_{2, \text { lin }} z /\left(T_{0}^{4}+\beta_{2, \text { lin }}{ }^{2} z^{2}\right)<0$ where $\beta_{2, \text { lin }}$ is the dispersion coefficient of the linear FBG [33]. Figure 5(c) shows the evolution of the normalized chirp $C_{\text {lin }}(z)$ in a linear grating for the Gaussian pulse. The maximum normalized chirp $\left|C_{\text {lin }}(z)\right|=0.5$ occurs at $z=L_{D}=T_{0}{ }^{2} / \beta_{2, \text { lin. }}$. Figure $5(\mathrm{~d})$ shows the evolution of the broadening factor until the maximum chirp occurs. The solid and dashed curves represent the broadening factor in the chirping of a hyperbolic secant and Gaussian pulse. The maximum broadening factors are 1.26 and 1.41 for a hyperbolic secant and Gaussian pulse.

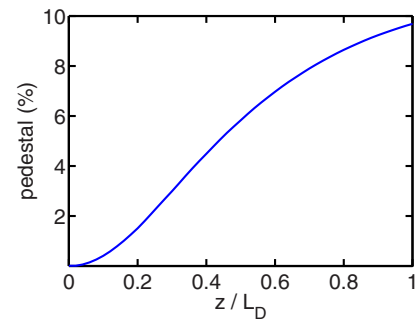

(a)

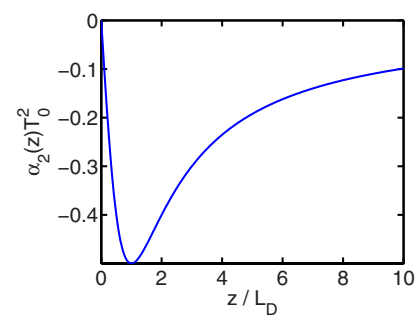

(c)

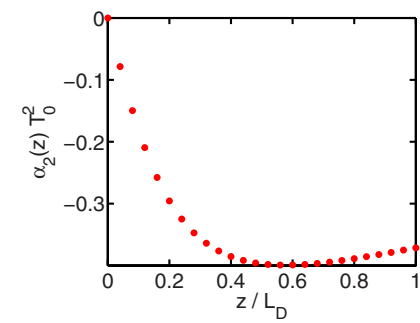

(b)



(d)
Fig. 5. (Color online) (a) Pedestal generated and (b) the evolution of $\alpha_{2}(z) T_{0}{ }^{2}$ in the chirping of a hyperbolic secant pulse using a linear FBG as a function of grating length. (c) The evolution of $\alpha_{2}(z) T_{0}^{2}$ in the chirping of a Gaussian pulse using a linear FBG versus grating length. (d) Evolution of the broadening factor until the maximum chirp occurs. The solid and dashed curves represent the broadening factor in the chirping of a hyperbolic secant and Gaussian pulse. The parameter $L_{D}$ is the dispersion length of the linear grating.
As an illustration, we consider the compression of a chirp-free hyperbolic secant pulse and a chirp-free Gaussian pulse with the same FWHM $16.65 \mathrm{ps}$, corresponding to $T_{0}=9.45 \mathrm{ps}$ for the hyperbolic secant pulse and $T_{0}$ $=10 \mathrm{ps}$ for the Gaussian pulse. For the hyperbolic secant input pulse, the dispersion coefficient and the length of the linear FBG are $25 \mathrm{ps}^{2} / \mathrm{cm}$ and $2 \mathrm{~cm}$, respectively. For the Gaussian input pulse, the dispersion coefficient and the length of the linear FBG are $25 \mathrm{ps}^{2} / \mathrm{cm}$ and $4 \mathrm{~cm}$, respectively. We use linear FBGs with the same dispersion but vary the grating length to achieve the maximum chirp value $\alpha_{20}$ in the chirping of the hyperbolic secant and Gaussian pulses. In our chosen examples, $\alpha_{20}$ is found to be $-0.0045 \mathrm{THz}^{2}$ for the prechirped quasi-hyperbolic secant pulse, and $-0.005 \mathrm{THz}^{2}$ for the prechirped Gaussian pulse.

\section{B. Nonlinear Compression}

Next, we launched the prechirped hyperbolic secant pulse, from Subsection 4.A, into the NFBG with exponentially decreasing dispersion. The power of the optical pulse input to the NFBG is chosen as $P_{0}$ $=\left|\beta_{20}\right| / \gamma_{g} / T_{\text {sech }}^{2}\left(L_{\mathrm{LFBG}}\right)$, where $T_{\text {sech }}\left(L_{\mathrm{LFBG}}\right)$ is the pulse width parameter of the hyperbolic secant pulse used to fit the linear FBG output when the input is a hyperbolic secant pulse, and $\beta_{20}$ is the initial dispersion value of the NFBG. The pulse width parameter of the prechirped pulse is $T_{\text {sech }}\left(L_{\mathrm{LFBG}}\right)=11.9 \mathrm{ps}$, the initial dispersion coefficient is $\beta_{20}=-25 \mathrm{ps}^{2} / \mathrm{cm}$, the decay rate of exponentially decreasing dispersion is $\sigma=0.1125 / \mathrm{cm}$, the nonlinear coefficient of NFBG is $\gamma_{\mathrm{g}}=15 \mathrm{~W} / \mathrm{km}$, and the length of NFBG is $19.2 \mathrm{~cm}$. Figures 6(a) and 6(b) show the initial pulse (solid curve) and pre-chirped pulse (dashed curve) in linear and logarithmic scales. We note that the chirped pulse deviates slightly from the hyperbolic secant profile. Figures 6(c) and 6(d) show the pulse profile after the NFBG $(19.2 \mathrm{~cm})$ in both linear and logarithmic scales. The fitted hyperbolic secant profile [dots in Fig. 6(c) and dashed curve in Fig. 6(d)] has the same peak power and the FWHM as the final compressed pulse. Figure 6(e) shows the evolution of the FWHM in the linear FBG (the prechirper) and the NFBG (the pulse compressor), where the dots, solid curve, dashed curve, and dotted-dashed curve represent self-similar soliton, the simulation results of NLS equation, the results of the LVM using hyperbolic secant pulse ansatz, and the results of the POM using hyperbolic secant pulse ansatz, respectively. The deviation is slightly larger here because the two reduction methods are not very accurate in determining the FWHM using the hyperbolic secant pulse ansatz in the prechirping process. We note that the LVM performs slightly better than the POM. The total compression ratio (compared to initially chirp-free pulse) is 6.28 , and the generated pedestal is $6.24 \%$ (Table 3 ).

We then launched the chirped Gaussian pulse, from Subsection 4.A, into the NFBG with exponentially decreasing dispersion. Following [34] we choose the parameters

$$
T_{\text {Gauss }}^{2}\left(L_{L F B G}\right) / \sqrt{2} /\left|\beta_{20}\right|=1 / \gamma_{g} / P_{0},
$$

where $T_{\text {Gauss }}\left(L_{L F B G}\right)$ is the pulse width parameter of the prechirped Gaussian pulse which is achieved after the lin- 




(a)

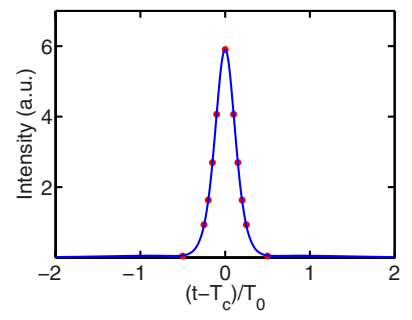

(c)

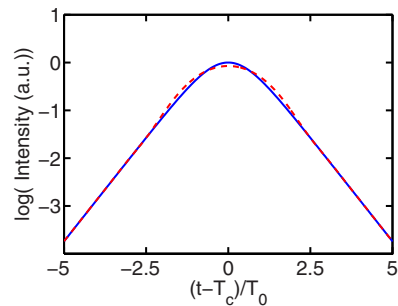

(b)

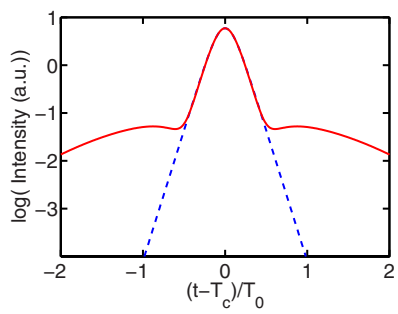

(d)

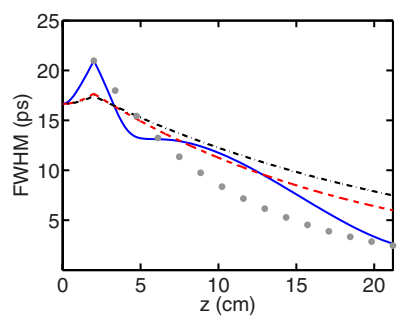

(e)

Fig. 6. (Color online) Hyperbolic secant input pulse. The pulse profiles before (solid curve) and after the linear FBG (dashed curve) in (a) linear and (b) logarithmic scales. The pulse profiles after the NFBG (solid curve) and a "fitted" hyperbolic secant pulse profiles in (c) linear (dots) and (d) logarithmic scales (dashed curve). (e) Evolution of the FWHM in the prechirper (the linear FBG) and the NFBG for the self-similar soliton (dots), the full numerical simulation (solid curve), the LVM using hyperbolic secant pulse ansatz (dashed curve), and the POM using hyperbolic secant pulse ansatz (dot-dashed curve).

ear FBG, $P_{0}$ is the power of pulse input to the NFBG, and $\beta_{20}$ is the initial dispersion value of the NFBG. In our example, $\quad \beta_{20}=-25 \mathrm{ps}^{2} / \mathrm{cm}, \quad T_{\text {Gauss }}\left(L_{L F B G}\right)=10 \sqrt{2} \mathrm{ps}, \quad \sigma$ $=0.125 / \mathrm{cm}, \gamma_{g}=15 \mathrm{~W} / \mathrm{km}$, and the NFBG is $16 \mathrm{~cm}$ long. Figures 7(a) and 7(b) show the initial pulse (solid curve) and prechirped pulse (dashed curve) in linear and logarithmic scales. Figures 7(c) and 7(d) show the pulse profile after the NFBG $(16 \mathrm{~cm})$ in both linear and logarithmic scales. From Fig. 7(d) the main portion of the compressed pulse is almost the same as the fitted hyperbolic secant



(a)

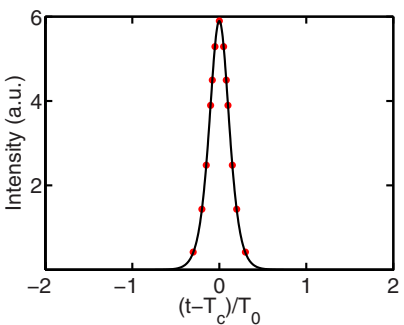

(c)

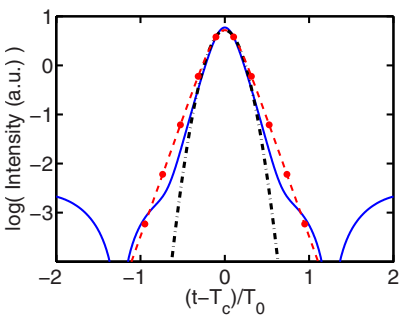

(e) (b)



(d)

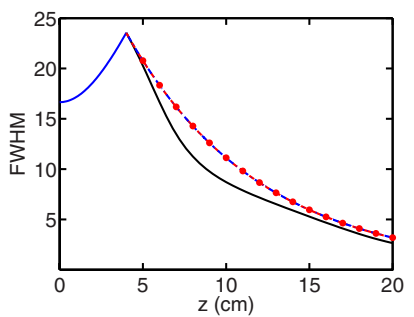

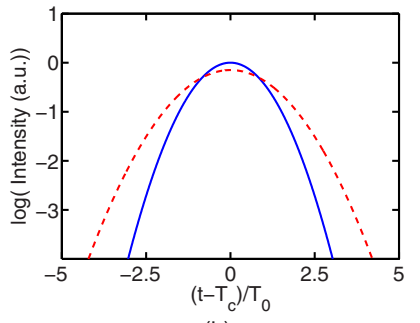

Fig. 7. (Color online) Gaussian input pulse. The pulse profiles before (solid curve) and after the linear FBG (dashed curve) in (a) linear and (b) logarithmic scales. The pulse profiles after the NFBG (solid curve) and a fitted hyperbolic secant pulse profiles in (c) linear (dots) and (d) logarithmic scales (dashed curve). (e) The compressed pulse in logarithmic scale. (f) Evolution of the FWHM in the prechirper (linear FBG) and the NFBG. For (e) and (f), the solid curve, dashed curve, dots, and dotted-dashed curve represent the full numerical simulation, the LVM with hyperbolic secant pulse ansatz, the POM with hyperbolic secant pulse ansatz, and the LVM (or POM) with Gaussian pulse ansatz, respectively.

pulse, indicating that the initial Gaussian profile has evolved into a hyperbolic secant pulse profile after the compression in the NFBG. For the compression of the Gaussian pulse, we solve the pulse parameters equations using the LVM and the POM using the hyperbolic secant pulse ansatz [Eq. (10)] and Gaussian pulse ansatz [Eq. (11)]. We assume that the pulse launched into the NFBG is chirped hyperbolic secant pulse or chirped Gaussian

\section{Table 3. Comparison Between the Compression of Hyperbolic Secant Input Pulse and Gaussian Input} Pulse $^{a}$

Compression of Hyperbolic Secant Input Pulse

(Fig. 6)

Compression of Gaussian Input Pulse (Fig. 7)

\begin{tabular}{lcc}
\hline$C(0)$ & -0.63 & -1 \\
$C(z)$ & -0.16 & -0.097 \\
FWHM of compressed pulse & $2.65 \mathrm{ps}$ & $2.65 \mathrm{ps}$ \\
Pedestal of compressed pulse & $6.24 \%$ & $0.0935 \%$
\end{tabular}

${ }^{a} C(0)$ is the normalized chirp coefficient of the chirped hyperbolic secant or Gaussian input pulse. The normalized chirp coefficients after the linear FBG [C(0)] are determind by fitting the phase of the pulse using $C(0) t^{2} / T^{2}\left(L_{\mathrm{LFBG}}\right) / 2$, where $T\left(L_{\mathrm{LFBG}}\right)$ is the pulse width parameter of the hyperbolic secant or Gaussian pulse. Similarly, the chirp coefficient of the compressed pulse, $C(z)$, is determined by fitting the phase of the pulse using $C(z) t^{2} / T^{2}\left(L_{\mathrm{NFBG}}\right) / 2$, where $T\left(L_{\mathrm{NFBG}}\right)$ is the pulse width parameter of the compressed pulse. 
pulse with same FWHM. Figure 7(e) shows the compressed pulse. Figure $7(\mathrm{f})$ shows the evolution of the FWHM in the linear FBG and NFBG. For both Figs. 7(e) and $7(\mathrm{f})$, the solid curve, dashed curve, dots, and dotteddashed curve represent the results from the full simulation, the LVM with hyperbolic secant pulse ansatz, the POM with hyperbolic secant pulse ansatz, and the LVM (or POM) with Gaussian pulse ansatz, respectively. The LVM with hyperbolic secant pulse ansatz approximates the full simulations results better. The total compression ratio when compared to the initial chirp-free pulse is 6.28 and the generated pedestal is $0.0935 \%$ (Table 3 ).

As shown in Table 3, the final compressed pulse of the initial hyperbolic secant and Gaussian pulses have the same FWHM (2.65 ps). The final compressed pulses are almost chirp-free. We observe that the pedestal generated from an input Gaussian pulse is much smaller than that of an input hyperbolic secant pulse. By varying $\beta_{20}, \alpha_{20}$ and $T_{0}$, we find that the final compression ratio mainly depends on the designed value of $\mathrm{CR}$, and the generated pedestal is associated with both the designed $\mathrm{CR}$ and $\varepsilon$ discussed before.

In the compression of initial Gaussian pulses, we use Eq. (15) to determine the NFBG parameters. We find that the design gives good quality compressed pulses. We want to determine whether Eq. (15) gives the optimal design parameters for the NFBG. We define the ratio $=L_{D 0, \text { Gauss }} / L_{N 0, \text { Gauss }}$, where $L_{D 0, \text { Gauss }}=T_{\text {Gauss }}^{2}\left(L_{L F B G}\right) /\left|\beta_{20}\right|$ and $L_{N 0, \text { Gauss }}=1 / \gamma_{\mathrm{g}} / P_{0}$ are the initial dispersion length and initial nonlinear length of the Gaussian pulse input into the NFBG. We obtain different ratios of $L_{D 0, \text { Gauss }} / L_{N 0, \text { Gauss }}$ by either changing the initial dispersion value of the NFBG $\left(\beta_{20}\right)$ or changing the peak power of the initial pulse. Different lengths of NFBG are used to achieve the same FWHM of the final compressed pulse. Table 4 gives the percentages of pedestals generated. We observe that the amount of pedestal generated mainly depends on the ratio $L_{D 0, \text { Gauss }} / L_{N 0 \text {,Gauss }}$ and the optimal point is $L_{D 0, \text { Gauss }} / L_{N 0, \text { Gauss }}=\sqrt{2}$. Figure 8 shows the evolution of the FWHM with respect to the change in the ratio $L_{D 0, \text { Gauss }} / L_{N 0, \text { Gauss. }}$ Thus Eq. (15) is a good and simple criterion to design the NFBG for compression of Gaussian pulses.

\section{Stepwise Approximation}

Finally, we consider the SWA of the NFBG used in the compression of an initial hyperbolic secant pulse dis-

Table 4. Comparison of the Pedestal Generated for Different Values of the Ratio $=L_{D 0, \text { Gauss }} / L_{N 0, \text { Gauss }}{ }^{a}$

\begin{tabular}{ccc}
\hline Ratio & Change of $\beta_{20}$ & Change of Peak Power \\
\hline 1 & $6.49 \%$ & $6.49 \%$ \\
1.2 & $1.47 \%$ & $1.47 \%$ \\
$\sqrt{2}$ & & $0.0935 \%$ \\
1.6 & $1 \%$ & \\
1.8 & $2.42 \%$ & $1 \%$ \\
2 & $3.69 \%$ & $2.38 \%$ \\
\hline
\end{tabular}

${ }^{a} L_{D 0, \text { Gauss }}=T_{\text {Gauss }}^{2}\left(L_{\mathrm{LFBG}}\right) /\left|\beta_{20}\right|$ and $L_{N 0, \text { Gauss }}=1 / \gamma_{g} / P_{0}$ are the initial dispersion and nonlinear lengths, respectively, of the ratio. The different values of $L_{D 0, \text { Gauss }} / L_{N 0 \text {,Gauss }}$ are obtained by either changing the initial dispersion value of NFBG $\beta_{20}$ or changing the peak power of the initial pulse. Different lengths of NFBG are used to achieve the same FWHM of the final compressed pulse.
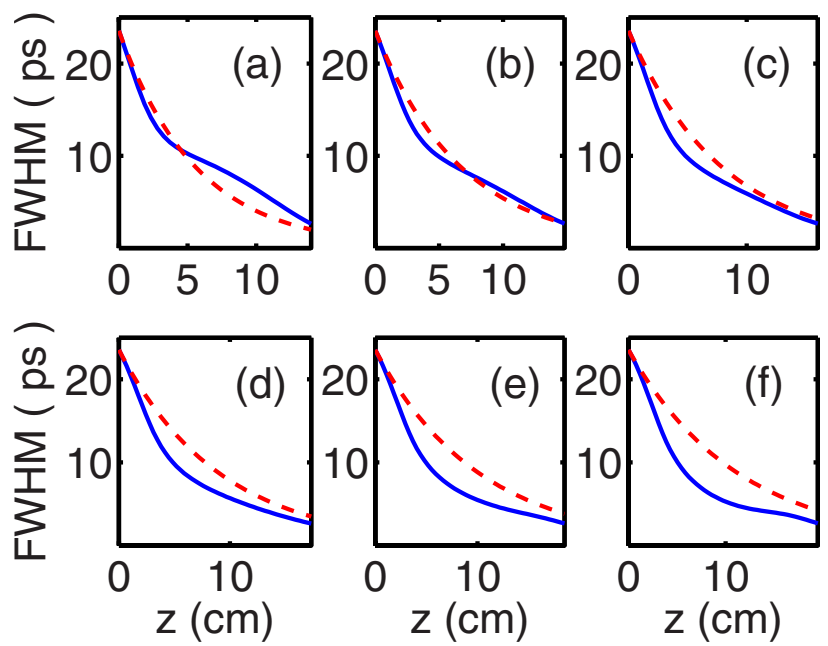

Fig. 8. (Color online) Evolution of the FWHM versus distance for different values of the ratio $L_{D 0, \text { Gauss }} / L_{N 0 \text {,Gauss. }}$ The dashed curve represents self-similar solitons and the solid curve represents simulation results. The values of the ratio $L_{D 0, \text { Gauss }} / L_{N 0, \text { Gauss }}$ from (a) to (f) are $1,1.2, \sqrt{2}, 1.6,1.8$, and 2 , respectively.

cussed in Subsection 4.A. Figures 9(a) and 9(b), respectively, show the change of FWHM and the percentage of pedestal generated of the final compressed pulse with respect to number of sections used. The dots represent the FWHM or the pedestal generated versus the number of sections used, and the circle represents that by using DDFBG. When the number of sections increases, the compressed pulse from the SWA becomes closer to that by using DDFBG. Figure 9(c) shows the evolution of the FWHM in the compression process, where the solid curve,

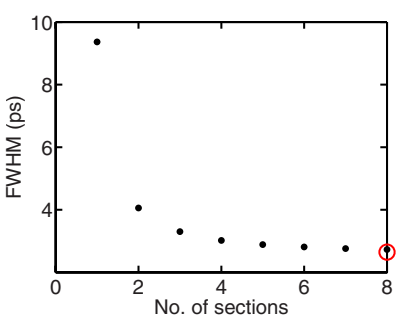

(a)



(b)

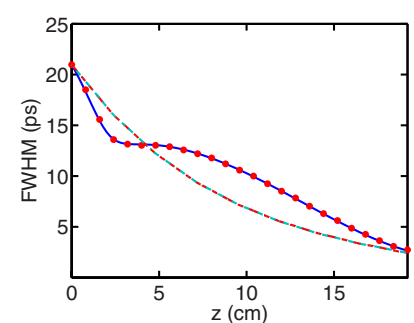

(c)

Fig. 9. (Color online) Hyperbolic secant input pulse and SWA to the exponentially decreasing dispersion. (a) The FWHM of the final compressed pulses using 1 to 8 sections for SWA (dots) and DDFBG (circle). (b) The pedestal generated in the final compressed pulse using 1 to 8 sections for SWA (dots) and DDFBG (circle). (c) Evolution of the FWHM in DDFBG (solid curve), the full numerical simulation of the eight-section SWA (dots), the LVM with hyperbolic secant pulse ansatz and the eight-section SWA (dashed curve), the POM with hyperbolic secant pulse ansatz, and the eight-section SWA (dotted-dashed curve). 
dots, dashed curve, and dotted-dashed curve represent the full simulation of DDFBG, the full simulation using an eight-section SWA, the results of the LVM using hyperbolic secant pulse ansatz and an eight-section SWA, and the results of the POM using hyperbolic secant pulse ansatz and an eight-section SWA, respectively. We observe that results from the LVM and POM using the hyperbolic secant pulse ansatz are very close to each other.

Next, we consider the SWA of the NFBG used in the compression of an initial Gaussian pulse discussed in the Subsection 4.A. Figures 10(a) and 10(b), respectively, show the change of the FWHM and the percentage of pedestal generated from the final compressed pulse versus the number of sections used. The dots represent the FWHM or the pedestal generated using different number of sections, and the circle represents that by using DDFBG. Again when the number of sections increases, the compressed pulse from the SWA becomes closer to that achieved in the DDFBG. Figure 10(c) shows the evolution of the FWHM in the eight-section SWA and DDFBG. In this case, eight FBGs with constant dispersions can approximate the exponentially decreasing dispersion profile very well. Finally Fig. 10(d) compares the results from the full simulation, the LVM and POM with hyperbolic secant pulse ansatz and Gaussian pulse ansatz. The dots, dashed curve, dotted-dashed curve, and solid curve represent full simulation of the eight-section SWA, the results of the LVM with hyperbolic secant pulse ansatz and the eight-section SWA, the results of the POM with hyperbolic secant pulse ansatz and the eight-section SWA, and the result of the LVM or POM with Gaussian

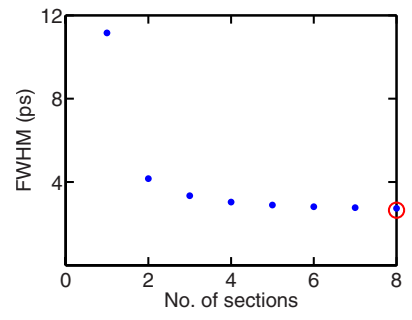

(a)

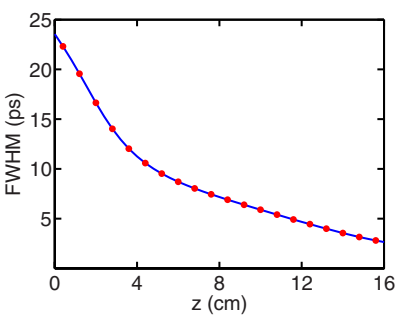

(c)

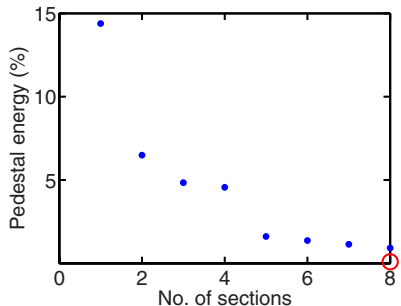

(b)

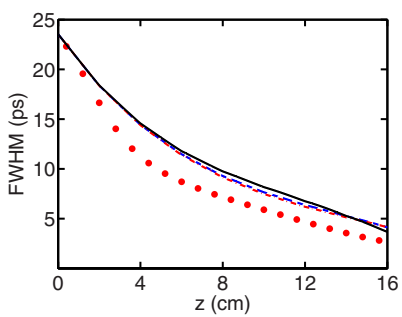

(d)
Fig. 10. (Color online) Gaussian input pulse and SWA to the exponentially decreasing dispersion. (a) Evolution of the FWHM of the final compressed pulse using 1 to 8 sections for SWA (dots) and DDFBG (circle). (b) The pedestal generated of the final compressed pulse using 1 to 8 sections for SWA (dots) and DDFBG (circle). (c) Evolution of the FWHM in DDFBG (solid curve) and the eight-section SWA using the full simulation (dots). (d) Evolution of the FWHM in the eight-section SWA for the full simulation on the eight-section SWA (dots), the LVM with hyperbolic secant pulse ansatz and the eight-section SWA (dashed curve), the POM with hyperbolic secant pulse ansatz and the eight-section SWA (dotted-dashed curve), and Gaussian pulse ansatz and the eight-section SWA (solid curve). pulse ansatz and the eight-section SWA, respectively. The results from the two reduction methods with different ansatz are similar and deviate slightly from that of full simulations.

\section{CONCLUSIONS}

In conclusion, we have shown that self-similar chirped Bragg soliton solutions for the nonlinear Schrödinger (NLS) equation exist for exponentially varying dispersion. The evolution of the pulse width follows that of the exponentially varying dispersion. Thus, nonlinear fiber Bragg gratings (FBGs) with exponentially decreasing dispersion can be used to carry out pedestal free compression of optical pulses. Unlike the solitons of the NLS equation with constant dispersion, these self-similar solitons are chirped. The chirped soliton evolves such that the dispersion length equals to the nonlinear length. Both the dispersion length and the nonlinear length vary with distance, but the chirp length is constant. As a result, if the ratio of the initial chirp length to the dispersion length is small, the characteristics of the pulse evolution will change from initially quasi-linear in the beginning of the grating, to a combination of linear and nonlinear for intermediate portion of the grating, to finally fully nonlinear towards the end of the grating.

We have also shown that the exponentially varying dispersion of the nonlinear FBGs can be approximated by using a concatenation of nonlinear FBGs (NFBGs) with different constant dispersions. For a given tolerance on the pedestal generated, we have found that the number of concatenated sections is proportional to the compression ratio, but inversely proportional to the initial chirp value. We have also studied the effect of prechirping using linear FBG to produce the required chirp profile for initially chirp-free hyperbolic secant and Gaussian pulse. We have found that the pedestal generated from an input Gaussian pulse is much smaller than that of a hyperbolic secant pulse showing that the compression by the nonlinear FBG is more sensitive to the chirp profile than the pulse shape. We have also found that the initial Gaussian profile evolves into a hyperbolic secant profile after the compression in the NFBG. Finally, we study the use of stepwise approximation (SWA) to the exponentially decreasing dispersion on initial hyperbolic secant and Gaussian pulses chirped by a linear FBG. We have found that high quality compressed pulses can be generated.

\section{ACKNOWLEDGMENTS}

The authors acknowledge the support of the Research Grant Council of the Hong Kong Special Administrative Region, China (project PolyU5289/07E).

\section{REFERENCES}

1. G. P. Agrawal, Applications of Nonlinear Fiber Optics (Academic, 2001).

2. R. H. Stolen, J. Botineau, and A. Ashkin, "Intensity discrimination of optical pulses with birefringent fibers," Opt. Lett. 7, 512-514 (1982). 
3. J. L. Tapié and G. Mourou, "Shaping of clean, femtosecond pulses at $1.053 \mu \mathrm{m}$ for chirped pulse amplification," Opt. Lett. 17, 136-138 (1992).

4. S. V. Chernikov and P. V. Mamyshev, "Femtosecond soliton propagation in fibers with slowly decreasing dispersion," J. Opt. Soc. Am. B 8, 1633-1641 (1991).

5. P. V. Mamyshev, S. V. Chernikov, and E. M. Dianov, "Generation of fundamental soliton trains for high-bit-rate optical fiber communication lines," IEEE J. Quantum Electron. 27, 2347-2355 (1991).

6. B. J. Eggleton, G. Lenz, and N. M. Litchinitser, "Optical pulse compression schemes that use nonlinear Bragg gratings," Fiber Integr. Opt. 19, 383-421 (2000).

7. S. V. Chernikov, J. R. Taylor, and R. Kashyap, "Experimental demonstration of steplike dispersion profiling in optical fibre for soliton pulse generation and compression," Electron. Lett. 30, 433-435 (1994).

8. J. D. Moores, "Nonlinear compression of chirped solitary waves with and without phase modulation," Opt. Lett. 21, $555-557$ (1996)

9. M. E. Fermann, V. I. Kruglov, B. C. Thomsen, J. M. Dudley, and J. D. Harvey, "Self-similar propagation and amplification of parabolic pulses in optical fibers," Phys. Rev. Lett. 84, 6010-6013 (2000).

10. V. I. Kruglov, A. C. Peacock, and J. D. Harvey, "Exact self-similar solutions of generalized nonlinear Schrödinger equation with distributed coefficients," Phys. Rev. Lett. 90, 113902 (2003).

11. V. I. Kruglov, A. C. Peacock, and J. D. Harvey, "Exact solutions of the generalized nonlinear Schrödinger equation with distributed coefficients," Phys. Rev. E 71, 056619 (2005).

12. C. Billet, J. M. Dudley, N. Joy, and J. C. Knight, "Intermediate asymptotic evolution and photonic bandgap fiber compression of optical similaritons around $1550 \mathrm{~nm}$," Opt. Express 13, 3236-3241 (2005).

13. N. M. Litchinitser, B. J. Eggleton, and D. B. Patterson, "Fiber Bragg gratings for dispersion compensation in transmission: theoretical model and design criteria for nearly ideal pulse recompression," J. Lightwave Technol. 15, 1303-1313 (1997).

14. B. J. Eggleton, R. E. Slusher, C. M. de Sterke, P. A. Krug, and J. E. Sipe, "Bragg grating solitons," Phys. Rev. Lett. 76, 1627-1630 (1996).

15. G. Lenz and B. J. Eggleton, "Adiabatic Bragg soliton compression in nonuniform grating structures," J. Opt. Soc. Am. B 15, 2979-2985 (1998).

16. K. Senthilnathan, P. K. A. Wai, and K. Nakkeeran, "Pedestal free pulse compression in nonuniform fiber Bragg gratings," in Proceedings of the Optical Fiber Communication Conference (2007), paper JWA19.

17. K. Senthilnathan, Q. Li, P. K. A. Wai, and K. Nakkeeran, "Bragg soliton pulse compression in non-uniform fiber Bragg gratings," in Proceedings of the OptoElectronics and Communications Conference (2007), paper 13C1-4.
18. Q. Li, P. K. A. Wai, K. Nakkeeran, and K. Senthilnathan, "Nearly chirp-free and pedestal-free pulse compression," in Proceedings of the OptoElectronics and Communications Conference (2007), paper 13P-21.

19. Q. Li, K. Senthilnathan, K. Nakkeeran, and P. K. A. Wai, "Pedestal-free pulse compression in nonlinear fiber Bragg gratings with exponentially varying dispersion," in Proceedings of the OptoElectronics and Communications Conference (2008), paper P18.

20. C. M. De Sterke and J. E. Sipe, "Gap solitons," Prog. Opt. 33, 203-260 (1994).

21. K. Senthilnathan and K. Porsezian, "Adiabatic Bragg soliton compression," Opt. Commun. 227, 275-281 (2003).

22. K. Senthilnathan, K. Nakkeeran, K. W. Chow, Q. Li, and P. K. A. Wai, "Chirped Optical Solitons," in Advances in Nonlinear Waves and Symbolic Computation (Nova, 2009).

23. E. N. Tsoy and C. M. de Sterke, "Propagation of nonlinear pulses in chirped fiber gratings," Phys. Rev. E 62, 2882-2890 (2000)

24. E. N. Tsoy and C. M. de Sterke, "Soliton dynamics in nonuniform fiber Bragg gratings," J. Opt. Soc. Am. B 18, 1-5 (2001).

25. A. Rosenthal and M. Horowitz, "Analysis and design of nonlinear fiber Bragg gratings and their application for optical compression of reflected pulses," Opt. Lett. 31, 1334-1336 (2006)

26. A. Rosenthal and M. Horowitz, "Bragg-soliton formation and pulse compression in one-dimensional periodic structure," Phys. Rev. E 74, 066611 (2006).

27. B. A. Malomed, "Variational methods in nonlinear fiber optics and related fields," Prog. Opt. 43, 69-191 (2002).

28. K. Nakkeeran and P. K. A. Wai, "Generalized projection operator method to derive the pulse parameters equations for the nonlinear Schrödinger equation," Opt. Commun. 244, 377-382 (2005).

29. P. Tchofo Dinda, A. B. Moubissi, and K. Nakkeeran, "Collective variable theory for optical solitons in fibers," Phys. Rev. E 64, 016608 (2001).

30. A. Hasegawa, S. Kumar, and Y. Kodama, "Reduction of collision-induced time jitters in dispersion-managed soliton transmission systems," Opt. Lett. 21, 39-41 (1996).

31. W.-h. Cao and P. K. A. Wai, "Picosecond soliton transmission by use of concatenated gain-distributed nonlinear amplifying fiber loop mirrors," Appl. Opt. 44, 7611-7620 (2005).

32. D. Grischkowsky and A. C. Balant, "Optical pulse compression based on enhanced frequency chirping," Appl. Phys. Lett. 41, 1-3 (1982).

33. G. P. Agrawal, Nonlinear Fiber Optics (Academic, 2001).

34. P. K. A. Wai and K. Nakkeeran, "On the uniqueness of Gaussian ansatz parameters equations: generalized projection operator method," Phys. Lett. A 332, 239-243 (2004). 